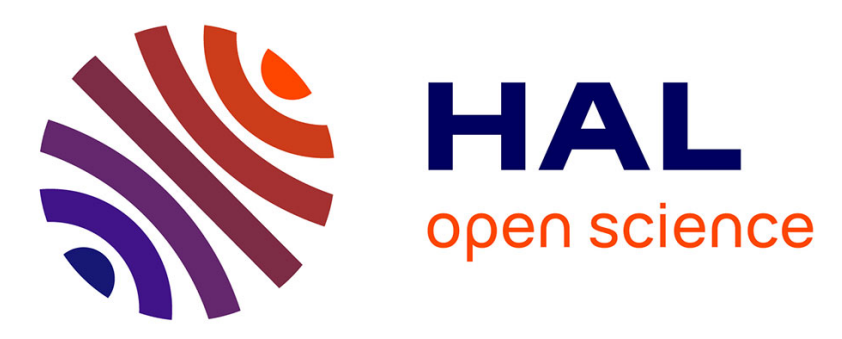

\title{
Rnd family genes are differentially regulated by 3,4-methylenedioxymethamphetamine and cocaine acute treatment in mice brain.
}

\author{
Cynthia Marie-Claire, Julie Salzmann, Alexandre David, Cindie Courtin, \\ Corinne Canestrelli, Florence Noble
}

\section{To cite this version:}

Cynthia Marie-Claire, Julie Salzmann, Alexandre David, Cindie Courtin, Corinne Canestrelli, et al.. Rnd family genes are differentially regulated by 3,4-methylenedioxymethamphetamine and cocaine acute treatment in mice brain.. Brain Research, 2007, 1134 (1), pp.12-7. 10.1016/j.brainres.2006.11.065 . inserm-00193078

\section{HAL Id: inserm-00193078 https://www.hal.inserm.fr/inserm-00193078}

Submitted on 30 Nov 2007

HAL is a multi-disciplinary open access archive for the deposit and dissemination of scientific research documents, whether they are published or not. The documents may come from teaching and research institutions in France or abroad, or from public or private research centers.
L'archive ouverte pluridisciplinaire HAL, est destinée au dépôt et à la diffusion de documents scientifiques de niveau recherche, publiés ou non, émanant des établissements d'enseignement et de recherche français ou étrangers, des laboratoires publics ou privés. 


\title{
Rnd family genes are differentially regulated by $3,4-$
} methylenedioxymethamphetamine and cocaine acute treatment in

\author{
mice brain
}

Cynthia Marie-Claire, Julie Salzmann, Alexandre David, Cindie Courtin,

Corinne Canestrelli, Florence Noble*

CNRS, UMR7157 ; INSERM, U705 ; Universite Paris Descartes, Neuropsychopharmacologie des addictions, Paris, F-75006 France

Number of pages : 18

Number of figures and tables: 4

* corresponding author

Tel : 33-1-53-73-95-61

Fax : 33-1-53-73-97-19

florence.noble@univ-paris5.fr 


\section{1- ABSTRACT}

Drugs of abuse induce alterations in cytoskeletal and cytoskeleton associated genes in several brain areas. We have previously shown that acute MDMA regulates the mRNA level of Rnd3, a Rho GTPase involved in actin cytoskeleton regulation, in mice striatum. In this study we investigated the effects of single administration of cocaine, another psychostimulant with a slightly different mechanism of action, on the mRNA levels of the three members of the Rnd genes family (Rnd1, Rnd2 and Rnd3). Mice were treated with either MDMA (9 mg/kg) and cocaine (20 mg/jg) and brain samples (i.e. hippocampus, striatum and prefrontal cortex) were processed for quantitative real-time PCR assay one, two, four and six hours after the injections. The expression level of Rnd2 was differentially affected depending on the drug, brain area and time point after injection. Interestingly the two drugs upregulate Rnd3 gene expression in the three structures tested with some differences in the timing. The effects of MDMA on Rnd3 appear earlier in the hippocampus as compared to cocaine, while it is the opposite in the prefrontal cortex. However in the dorsal striatum the two drugs induce an early and significant upregulation of Rnd3 expression that is longer-lasting in the case of MDMA. In the case of cocaine contrarily to what was observed with MDMA this modulation could not be blocked with the ERK activation inhibitor SL327 suggesting that the two drugs lead to the same effect on Rnd3 by two distinct pathways.

Section 1 : Cellular and Molecular Biology of Nervous Systems

Keywords : cocaine, Rnd3, MDMA, gene expression, acute 
Abbreviations : PCR, polymerase chain reaction ; DMSO, dimethyl sulfoxide; MDMA: 3,4methylenedioxymethamphetamine 


\section{2-INTRODUCTION}

A large body of literature indicates that the expression and function of cytoskeleton-related proteins are altered by either acute or chronic morphine in rodents (Beitner-Johnson et al., 1992; Loguinov et al., 2001; Salzmann et al., 2004). Some of these proteins, like the neurofilaments, are also altered in postmortem brains of human opioid addicts (Ferrer-Alcon et al., 2000; Garcia-Sevilla et al., 1997). Interestingly, neurofilaments are also altered, in rats, by another drug of abuse with a completely different mechanism of action : cocaine (BeitnerJohnson et al., 1992). Recently, Ferrario and co-workers demonstrated that some of these alterations may have a functional significance as the increase in the density of dentritic spines observed in the nucleus accumbens of rats self-administering cocaine was associated with the transition from stable to escalated use of cocaine (Ferrario and Robinson). Moreover, a microarray study of prefrontal cortex of human cocaine abusers has also shown a regulation of neurofilaments and other cytoskeleton-related proteins by this drug (Lehrmann et al., 2003).

Recently, using oligonucleotide arrays, we identified several genes regulated by acute administration of the substituted amphetamine MDMA through ERK-dependent pathway within mice dorsal striatum (Salzmann et al., 2006). Among the genes identified in this study Rnd3 was found up-regulated and this modulation was partially dependent on ERK activation (Salzmann et al., 2006). Rnd3 also known as Rhoe or Arhe belongs to the Rnd subfamily of small Rho GTPases (Riento et al., 2005). This family plays a major role in actin cytoskeleton modulation and cell adhesion. The three members of the family (Rnd1, Rnd2 and Rnd3) are unusual in that they do not hydrolyse GTP (review in (Chardin, 2003). Thus, Rnd proteins would not be regulated by GTPase-activating proteins, instead they can be considered as constitutively active, and might be controlled mainly through transcription modulation. Rnd1 and Rnd 2 are mainly expressed in brain, whereas Rnd3 is expressed ubiquitously (Foster et 
al., 1996; Guasch et al., 1998; Nobes et al., 1998). Rnd1 has been shown to promote spine maturation (Ishikawa et al., 2003) and Rnd2 to stimulate dendrite branching (Fujita et al., 2002). As demonstrated in several cellular models, Rnd3 regulates actin cytoskeleton organization and cell migration (Guasch et al., 1998; Riento and Ridley, 2003).

We therefore investigated the regulation of the three Rnd genes by MDMA in order to determine if all the members of the Rnd family could be regulated by this drug. We also studied the modulation of these genes by another psychostimulant able to alter neuronal cytoskeleton : cocaine. These two drugs of abuse share the same biological targets but have distinct mechanisms of action. Cocaine's rewarding effects can be fully explained by the blockade of dopamine and serotonin transporter (DAT and SERT) (Hall et al., 2004). On the contrary, MDMA's mechanism of action is complex and not well-known. An inhibition of DAT and SERT has been proposed but the drug also displays a moderate affinity to a broad variety of receptors whose activation could be at the origin of certain of its effects (Battaglia et al., 1988; Colado et al., 2004). We characterized the patterns of Rnd genes expression in the prefrontal cortex, hippocampus, and striatum of mice, brain regions well known to play a critical role in the action of psychoactive drugs. Furthermore the regulation of the Rnd genes was investigated at two and four hours after the injection of the drugs in order to analyze the kinetics of the observed regulations. 


\section{3-RESULTS}

\section{Prefrontal cortex}

As compared to the controls, acute treatment with cocaine and MDMA had no effect on the mRNA levels of the three tested proteins in the prefrontal cortex during the first four hours after the injection (figure 1). Four hours after the injection a 1.61 fold increase of the transcription of Rnd2 and a 1.78 fold increase in that of Rnd3 was observed in response to cocaine treatment. In this structure among the three Rnd genes only Rnd3 showed a late modulation by MDMA six hours after the injection (1.38 fold increase).

\section{Hippocampus}

The mRNA levels of Rnd1 and Rnd2 were not affected by the two drugs in this structure at none of the tested time points (figure 2. Four hours after the injection Rnd3 mRNA was increased by 1.5 fold by MDMA and this modulation was still significant after six hours (1.24 fold). In the case of cocaine treatment only Rnd3 mRNA was found modulated in this structure six hours after the injection (1.24 fold).

\section{Striatum}

Rnd2 level of expression was not affected by the two tested drugs in this structure. As shown on figure 3 a slight 0.8 fold modulation of Rnd1 mRNA was observed in the dorsal striatum one hour after cocaine injection while the transcriptions of this gene was not affected by MDMA. As observed in the other structures Rnd3 is the only gene modulated by the two drugs. One hour after the injection the mRNA level of Rnd3 was significantly increased in this structure by cocaine (2.04 fold) and MDMA (2.58 fold). This upregulation was still significant two hours after the injection of cocaine and MDMA by 1.6 fold and 2.6 fold respectively. The marked increase observed with MDMA persisted at four and six hours after the injection (1.7 and 1.4 fold respectively) while the mRNA levels of Rnd3 returned to basal with cocaine. 
In order to determine whether the modulation of Rnd3 by the two drugs depend on the same pathways mice were pretreated with the inhibitor of ERK phosphorylation SL327. We have previously shown that $85 \%$ of the MDMA-induced Rnd3 up-regulation, at two hours after the injection, is inhibited by SL327 (Salzmann et al., 2006). In this study we found that the cocaine-induced Rnd3 regulation was not affected by a pretreatment with the same inhibitor of ERK pathway (Figure 4). 


\section{4-DISCUSSION}

In our previous study using a microarray approach we identified Rnd3, a small Rho GTPase involved in the regulation of actin cytoskeleton, among the genes regulated by acute MDMA in mice striatum (Salzmann et al., 2006). In order to extend this finding we performed acute injections of two psychostimulants cocaine and MDMA to test whether these drugs with similar but different mechanisms of action could alter the expression levels of the three members of the Rnd family (Rnd1, 2 and 3) in several brain structures at four time points after injection. The brain structures (striatum, prefrontal cortex and hippocampus) were selected based on their implication in important behaviors for addiction (Gerdeman et al., 2003; Kelley, 2004). The specificity of the regulations observed might be explained by the differences in the promoter regions of these three genes. An analysis of the promoters of Rnd1, 2 and 3 with MatInspector ${ }^{\circledR}$ software showed that although they share twenty putative transcription factor binding sites their number and localization vary. The results obtained indicate that these closely related GTPases are not functionally redundant for the effects of the two drugs of abuse tested.

Among the three proteins of the Rnd family two are highly expressed in brain. A role of Rnd1 in spine formation has been shown in cultured hippocampal neurons (Ishikawa et al., 2003). The mRNA level of this protein was modulated at only one time point by MDMA in the dorsal striatum in our conditions. Suggesting that the impact of Rnd1 on the changes induced in neuronal plasticity by acute administration of MDMA is probably negligible.

In the brain, Rnd2 is mainly expressed in the neurons of the hippocampus and cerebellum (Decourt et al., 2005; Nishi et al., 1999). Rnd2 mRNA level was not affected by the injection of the two tested drugs in the hippocampus and the striatum. On the contrary Rnd 2 was specifically up-regulated by cocaine in the prefrontal cortex four hours after the injection. Rnd2 is specifically expressed in neurons (Foster et al., 1996). An involvement of Rnd2 in 
neurite branching in PC12 cells has been demonstrated (Fujita et al., 2002). The effect observed here in the prefrontal cortex suggest a stimulation of neurite branching in the case of cocaine in this structure.

The mRNA level of Rnd3 was affected by the two drugs tested in the three structures at different levels and time post-injection. These results suggest that Rnd3 is a common effector of cocaine and MDMA. This study confirmed the up-regulation of Rnd3 in mice striatum two hours after acute injection of MDMA (Salzmann et al., 2006) and extend this modulation to another drug of abuse : cocaine. The striatum is the structure in which the modulations observed are the earliest, the most important and durable. This could be due to the greater endogenous dopamine content in this structure as compared to the prefrontal cortex and hippocampus (Míguez et al., 1999). In line with this, the striatum is the only brain structure tested in which morphine, another drug of abuse with a distinct mechanism of action, upregulates Rnd3 two hours after a single $10 \mathrm{mg} / \mathrm{kg}$ intraperitoneal injection (data not shown). The observed increase of Rnd3 mRNA could thus result from the release of dopamine induced by the drugs in this structure. Another possible explanation could be that one of the critical protein (receptor, signal transduction component...) for the up-regulation of Rnd3 is expressed at a very low level in the two other structures as compared to the striatum leading to the observed delayed effect.

The regulations of Rnd3 by cocaine and MDMA in the dorsal striatum follow descending kinetics. Rnd3 mRNA level remained significantly up-regulated six hours after MDMA treatment while its modulation by cocaine returned to basal four hours after the injection. This delay could result from distinct cellular mechanisms mediating gene expression. Therefore, in contrast to what was previously observed with MDMA (Salzmann et al., 2006), the inhibition of ERK phosphorylation by SL327 had no effect on the up-regulation of Rnd3 induced by cocaine. After MDMA repeated administration, Rnd3 mRNA levels were similar in the 
striatum of the saline and treated groups indicating that the modulation observed here was not persistent (data not shown).

Over-expression of Rnd proteins causes disruption of actomyosin contractile fibers and cell rounding (review in (Chardin, 2003). In neurons it has become largely accepted that RhoA inhibits neurite outgrowth (Luo et al., 1996). Rnd proteins counteract the biological functions of RhoA thus promoting neurite and/or dendrite outgrowth. Two main mechanisms for this inhibition have been suggested in the case of the most well known member of the family. Rnd3 inhibits RhoA function by interacting with p190RhoGAP, a GAP (GTPase-activating protein) for RhoA (Wennerberg et al., 2003). Rnd3 also antagonizes RhoA by interacting with one of the most important Rho effectors RockI which is known to stimulate actin reorganization by phosphorylating several actin-associated proteins (Riento et al., 2003; Riento and Ridley, 2003). A role of Rnd3 in cell cycle progression and transformation has also been described (Bektic et al., 2005; Riento et al., 2005). Since the Rnd proteins activities are mainly regulated through the balance between transcription-translation and degradation, an increase in the mRNA level of these genes is likely to have a real biological significance.

An increase in spine density and dendritic branching has been observed in the prefrontal cortex after repeated exposure to cocaine (Robinson and Kolb, 1999). The cocaine-induced modulations of Rnd2 and Rnd3 observed here in this structure might play an initiator role in the modifications observed. However, Rnd proteins do not seem to play a major role in the neural plasticity induced by MDMA in the prefrontal cortex. MDMA unlike cocaine has similar affinities for a broad range of receptors and monoamine transporters (Colado et al., 2004). Therefore the different patterns of Rnd genes modulations observed in each structure could be attributed to the different patterns of cellular pathways that can be activated by the two drugs. The striatum is the tested brain structures in which the effects of the two drugs were the most important, the modulation observed is transient and independent from ERK 
pathway in the case of cocaine but persistent and dependent on ERK activation in that of MDMA. These results suggest that although the two drugs share common cellular targets (DAT and SERT) and may activate the same signalling pathway in this structure (ERK pathway) their effects on a specific gene can be mediated by distinct mechanisms. Furthermore, alterations of the Rho GTPase pathway and subsequently neural plasticity induced in the striatum might be more pronounced in the case of MDMA.

The modulation of Rnd genes by cocaine and MDMA in mice striatum suggest modification of the dendritic branching and neurite outgrowth. In the case of cocaine a positive correlation between changes induced in spine density in the core of the nucleus accumbens and behavioral sensitization induced by the drug has been demonstrated (Li et al., 2004). It would be interesting to study if the same relationships exist in the case of MDMA. 


\section{EXPERIMENTAL PROCEDURE}

\section{Animals and drugs}

Male CD-1 mice (Charles River, France) weighing 22-24 g were housed in a room with $12 \mathrm{~h}$ alternating light/dark cycle and controlled temperature $\left(21 \pm 1^{\circ} \mathrm{C}\right)$. Food and water were available ad libitum. All drugs were injected intraperitoneally (i.p.). Cocaine (Sigma, France) and MDMA (Lipomed, Switzerland) were dissolved in saline solution $(0.9 \% \mathrm{NaCl})$. The MEK inhibitor SL327, a generous gift of Bristol-Myers Squibb (Wilmington) was dissolved in 100\% DMSO, as previously described (Selcher et al., 1999). Volumes of injection were 0.1 $\mathrm{ml}$ and $0.02 \mathrm{ml}$ per $10 \mathrm{~g}$ of body weight for MDMA (or saline) and SL327 (or vehicule), respectively. All animals received two injections.

\section{Drug treatment and dissection}

SL327 (50 mg/kg i.p.) was injected one hour before cocaine and MDMA as previously described (Salzmann et al., 2003). The doses of MDMA (9 mg/kg ; i.p.) and cocaine (20 $\mathrm{mg} / \mathrm{kg}$; i.p.) were chosen based on previous studies showing an activation of ERK, hyperlocomotion and place preference in mice (Anderson and Itzhak, 2003; Salzmann et al., 2003; Valjent et al., 2004). Mice were killed by cervical dislocation at one, two, four or six hours after the last injection. The brain was quickly removed, frozen in isopentane at $-50^{\circ} \mathrm{C}$, and placed in an acrylic matrice (David Kopf Instruments, Phymep, France) allowing the reproducible slicing of $1 \mathrm{~mm}$ coronal sections. According to The Mouse Brain Paxinos and Franklin Atlas (Academic Press, $2^{\text {nd }}$ edition, 2001) prefrontal cortex, hippocampus and dorsal striatum were then dissected free-hand on ice within the slices, and stored at $-80{ }^{\circ} \mathrm{C}$ until processing. 


\section{RNA isolation and Reverse Transcription for quantitative PCR}

Total RNA used for quantitative PCR experiments were extracted by a modified acidphenol guanidinum method, following the manufacturer's protocol (RNABle ${ }^{\circledR}$, Eurobio, France). The quality of the RNA samples was determined by electrophoresis through agarose gels and staining with ethidium bromide. Quantification of total RNA was assessed using a NanoDrop® ${ }^{\circledR}$ ND-1000 spectrophotometer (NanoDrop® ${ }^{\circledR}$ Technologies, USA). Reverse transcription of RNA was performed in a final volume of $20 \mu 1$ containing 1x first strand buffer (Invitrogen, France), $500 \mu \mathrm{M}$ each dNTP, $20 \mathrm{U}$ of Rnasin ribonuclease inhibitor (Promega, France), $10 \mathrm{mM}$ dithiothreitol, $100 \mathrm{U}$ of Superscript II Rnase $\mathrm{H}^{-}$reverse transcriptase (Invitrogen, France), $1.5 \mu \mathrm{M}$ random hexanucleotide primers (Amersham Biosciences, France) and $1 \mu \mathrm{g}$ of total RNA as previously described (Salzmann et al., 2003).

\section{Real-time quantitative RT-PCR}

PCR primers were chosen with the assistance of Oligo 6.42 software (MedProbe, Norway). Sequences of the primers used were as follow : Rndl forward 5'-cagccgtccagagacc3'; Rndl reverse 5'-gcagcaataagcaaaacac-3'; Rnd2 forward 5'-ccetggctggaaggtcag-3' and Rnd2 reverse 5'-tggggtgaggagtgacagc-3'. The primer nucleotide sequences used for $\mathrm{Hprt}$ and Rnd3 have been previously described (Salzmann et al., 2006). Fluorescent PCR reactions were performed on a Light-Cycler® instrument (Roche Diagnostics, Meylan, France) using the LC-FastStart DNA Master ${ }^{\text {plus }}$ SYBR Green I kit (Roche Diagnostics, Meylan, France). The cDNAs were diluted 500-fold and $5 \mu 1$ were added to the PCR reaction mix to yield a total volume of $10 \mu \mathrm{l}$. The reaction buffer contained $0.5 \mu \mathrm{M}$ of each primer. The PCR reactions were performed with 12 samples/drug treatment, each sample being prepared with bilateral structures from one mouse. Quantification was made on the basis of a calibration curve using cDNA from an untreated mouse brain. In addition to the genes of interest, the Hprt transcript (hypoxanthine guanine phosphoribosyl transferase) was also quantified and 
each sample was normalized on the basis of its Hprt content (as previously described (Salzmann et al., 2006). Fold change represent the ratio between (gene of interest transcript / Hprt transcript) cocaine or MDMA (gene of interest transcript / Hprt transcript $)_{\text {saline }}$ at each time point.

\section{Statistical analysis}

All series of data were analysed with GraphPad Prism ${ }^{\circledR} 4.0$ software. Statistical analyses were performed using one-way ANOVA between subjects, followed by Dunnett or Bonferroni tests for post-hoc comparisons. The level of significance was set at $\mathrm{p}<0.05$. 


\section{ACKNOWLEDGMENTS}

Julie Salzmann was supported by a fellowship from the Chancellery of the Universities of Paris. The authors thank Didier Fauconnier for helpful technical assistance. 


\section{REFERENCES}

Anderson, K. L., Itzhak, Y., 2003. Inhibition of neuronal nitric oxide synthase suppresses the maintenance but not the induction of psychomotor sensitization to MDMA ('Ecstasy') and p-chloroamphetamine in mice. Nitric Oxide. 9, 24-32.

Battaglia, G., et al., 1988. MDMA-induced neurotoxicity: parameters of degeneration and recovery of brain serotonin neurons. Pharmacol Biochem Behav. 29, 269-74.

Beitner-Johnson, D., et al., 1992. Neurofilament proteins and the mesolimbic dopamine system: common regulation by chronic morphine and chronic cocaine in the rat ventral tegmental area. J Neurosci. 12, 2165-76.

Bektic, J., et al., 2005. Small G-protein RhoE is underexpressed in prostate cancer and induces cell cycle arrest and apoptosis. Prostate. 64, 332-40.

Chardin, P., 2003. GTPase regulation: getting aRnd Rock and Rho inhibition. Curr Biol. 13, R702-4.

Colado, M. I., et al., 2004. Acute and long-term effects of MDMA on cerebral dopamine biochemistry and function. Psychopharmacology (Berl). 173, 249-63.

Decourt, B., et al., 2005. Expression analysis of neuroleukin, calmodulin, cortactin, and Rho7/Rnd2 in the intact and injured mouse brain. Brain Res Dev Brain Res. 159, 3654.

Ferrario, C. R., Robinson, T. E., Amphetamine pretreatment accelerates the subsequent escalation of cocaine self-administration behavior. European Neuropsychopharmacology. In Press, Corrected Proof.

Ferrer-Alcon, M., et al., 2000. Regulation of nonphosphorylated and phosphorylated forms of neurofilament proteins in the prefrontal cortex of human opioid addicts. J Neurosci Res. 61, 338-49.

Foster, R., et al., 1996. Identification of a novel human Rho protein with unusual properties: GTPase deficiency and in vivo farnesylation. Mol. Cell. Biol. 16, 2689-2699.

Fujita, H., et al., 2002. Rapostlin Is a Novel Effector of Rnd2 GTPase Inducing Neurite Branching. J. Biol. Chem. 277, 45428-45434.

Garcia-Sevilla, J. A., et al., 1997. Marked decrease of immunolabelled 68 kDa neurofilament (NF-L) proteins in brains of opiate addicts. Neuroreport. 8, 1561-5.

Gerdeman, G. L., et al., 2003. It could be habit forming: drugs of abuse and striatal synaptic plasticity. Trends Neurosci. 26, 184-92.

Guasch, R. M., et al., 1998. RhoE Regulates Actin Cytoskeleton Organization and Cell Migration. Mol. Cell. Biol. 18, 4761-4771.

Hall, F. S., et al., 2004. Molecular Mechanisms Underlying the Rewarding Effects of Cocaine. Ann NY Acad Sci. 1025, 47-56.

Ishikawa, Y., et al., 2003. A Role of Rnd1 GTPase in Dendritic Spine Formation in Hippocampal Neurons. J. Neurosci. 23, 11065-11072.

Kelley, A. E., 2004. Memory and Addiction: Shared Neural Circuitry and Molecular Mechanisms. Neuron. 44, 161-179.

Lehrmann, E., et al., 2003. Transcriptional profiling in the human prefrontal cortex: evidence for two activational states associated with cocaine abuse. Pharmacogenomics J. 3, 27 40.

Li, Y., et al., 2004. The induction of behavioural sensitization is associated with cocaineinduced structural plasticity in the core (but not shell) of the nucleus accumbens. European Journal of Neuroscience. 20, 1647-1654.

Loguinov, A. V., et al., 2001. Gene expression following acute morphine administration. Physiol Genomics. 6, 169-81. 
Luo, L., et al., 1996. Differential effects of the Rac GTPase on Purkinje cell axons and dendritic trunks and spines. Nature. 379, 837-40.

Míguez, J. M., et al., 1999. Selective changes in the contents of noradrenaline, dopamine and serotonin in rat brain areas during aging. Journal of Neural Transmission. V106, 10891098.

Nishi, M., et al., 1999. RhoN, a novel small GTP-binding protein expressed predominantly in neurons and hepatic stellate cells. Brain Res Mol Brain Res. 67, 74-81.

Nobes, C. D., et al., 1998. A New Member of the Rho Family, Rnd1, Promotes Disassembly of Actin Filament Structures and Loss of Cell Adhesion. J. Cell Biol. 141, 187-197.

Riento, K., et al., 2003. RhoE Binds to ROCK I and Inhibits Downstream Signaling. Mol. Cell. Biol. 23, 4219-4229.

Riento, K., Ridley, A. J., 2003. Rocks: multifunctional kinases in cell behaviour. Nat Rev Mol Cell Biol. 4, 446-56.

Riento, K., et al., 2005. Function and regulation of RhoE. Biochem Soc Trans. 33, 649-51.

Robinson, T. E., Kolb, B., 1999. Alterations in the morphology of dendrites and dendritic spines in the nucleus accumbens and prefrontal cortex following repeated treatment with amphetamine or cocaine. The European Journal Of Neuroscience. 11, 1598-1604.

Salzmann, J., et al., 2006. Analysis of transcriptional responses in the mouse dorsal striatum following acute 3,4-methylenedioxymethamphetamine (ecstasy): Identification of extracellular signal-regulated kinase-controlled genes. Neuroscience. 137, 473-482.

Salzmann, J., et al., 2003. Importance of ERK activation in behavioral and biochemical effects induced by MDMA in mice. Br J Pharmacol. 140, 831-8.

Salzmann, J., et al., 2004. [Acute and long-term effects of ecstasy]. Presse Med. 33, 24-32.

Selcher, J. C., et al., 1999. A necessity for MAP kinase activation in mammalian spatial learning. Learn Mem. 6, 478-90.

Valjent, E., et al., 2004. Addictive and non-addictive drugs induce distinct and specific patterns of ERK activation in mouse brain. Eur J Neurosci. 19, 1826-1836.

Wennerberg, K., et al., 2003. Rnd proteins function as RhoA antagonists by activating p190 RhoGAP. Curr Biol. 13, 1106-15. 
Figure 1 : Kinetics of the regulation of Rnd genes by cocaine $(20 \mathrm{mg} / \mathrm{kg})$ and MDMA (9 $\mathrm{mg} / \mathrm{kg}$ ) in mice prefrontal cortex. Results represent the fold change of the treated group as compared to the saline group (10-12 animals per group). Statistical analysis was done by ANOVA followed by Dunnet test. \#\# $\mathrm{P}<0.01$ for Rnd2 and ** $\mathrm{P}<0.01$ for Rnd3.

Figure 2 : Kinetics of the regulation of Rnd genes by cocaine $(20 \mathrm{mg} / \mathrm{kg})$ and MDMA (9 $\mathrm{mg} / \mathrm{kg}$ ) in mice hippocampus. Results represent the fold change of the treated group as compared to the saline group (10-12 animals per group). Statistical analysis was done by ANOVA followed by Dunnet test.; ${ }^{*} \mathrm{P}<0.05$, $* * \mathrm{P}<0.01$ for Rnd3.

Figure 3 : Kinetics of the regulation of Rnd genes by cocaine $(20 \mathrm{mg} / \mathrm{kg})$ and MDMA (9 $\mathrm{mg} / \mathrm{kg}$ ) in mice striatum. Results represent the fold change of the treated group as compared to the saline group (10-12 animals per group). Statistical analysis was done by ANOVA followed by Dunnet test. $\S \S \mathrm{P}<0.01$ for Rnd1; and $* \mathrm{P}<0.05$, ** $\mathrm{P}<0.01$ for Rnd3.

Figure 4 : Effect of SL327 (50mg/kg) pre-treatment on the regulation of Rnd3 by cocaine $(20 \mathrm{mg} / \mathrm{kg}$ ) in mice striatum. SL327 was administered $1 \mathrm{~h}$ before the drugs and mice were killed 2 hours after. Results represent the means \pm SEM (10-12 animals per group). Statistical analysis was done by ANOVA followed by Bonferroni test. * $\mathrm{P}<0.05$, ** $\mathrm{P}<0.01$ as compared to the control group. 